\title{
Depressionen bei Patienten mit Lungenkrebs
}

\author{
Patienten mit Lungenkrebs zeigen \\ häufig Symptome einer Depression. \\ Welchen Einfluss diese auf das Über- \\ leben haben, wurde nun untersucht.
}

\begin{abstract}
n dieser prospektiven Beobachtungsstudie des Cancer Care Outcomes Research and Surveillance Consortiums der USA wurden 1.790 Patienten innerhalb von 3 Monaten nachdem sie die $\mathrm{Di}$ agnose Lungenkrebs erhalten hatten in die Studie aufgenommen. $57 \%$ von ihnen waren über 65 Jahre alt, $45 \%$ waren Frauen. Um Depressionen zu erfassen, wurde die 8-Item-CES-D(Center for Epidemiologic Studies Depression)-Skala zum Zeitpunkt der Diagnose und nach 12 Monaten angewendet.

$38 \%$ der Studienteilnehmer hatten zu Studienbeginn Symptome einer Depression, weitere $14 \%$ entwickelten solche im Verlauf der Therapie. Die Patienten wurden basierend auf ihrer depressiven Symptomatik in 1 von 4 Gruppen eingeteilt: Patienten, die niemals Symptome einer Depression hatten $(n=640)$, Patienten mit erstmaliger Depressionssymp-
\end{abstract}

tomatik $(\mathrm{n}=105)$, Patienten mit Remission ihrer Depressionssymptomatik ( $\mathrm{n}=156)$ und Patienten mit persistierenden Symptomen einer Depression $(\mathrm{n}=254)$. Die Gruppe der Patienten, die niemals Symptome einer Depression zeigten, galt als Referenzgruppe.

In zwei Gruppen bestand ein erhöhtes Sterberisiko: Die Hazard Ratios für die Mortalität betrugen 1,50 ( $\mathrm{p}=0,006)$ bei Patienten mit erstmaliger Depressionssymptomatik und $1,42(\mathrm{p}=0,001)$ bei Patienten mit persistierender Depressionssymptomatik. Bei Patienten mit Remission ihrer Depressionssymptomatik lag keine erhöhte Mortalität vor (HR 1,02; $p=0,89$ ). Symptome einer Depression zu Studienbeginn waren bei Patienten mit Tumoren im frühen Stadium (I und II) mit einer erhöhten Mortalität assoziiert (HR 1,61; $\mathrm{p}<0,001$ ), nicht aber bei Patienten mit Tumoren in späten Stadien (III und IV; HR 1,05; $\mathrm{p}=0,45$ ).

Symptome einer Depression beim Follow-up nach 12 Monaten waren bei Patienten mit Tumoren in frühen Stadien (HR 1,71; $<<0,001)$ sowie bei Patienten

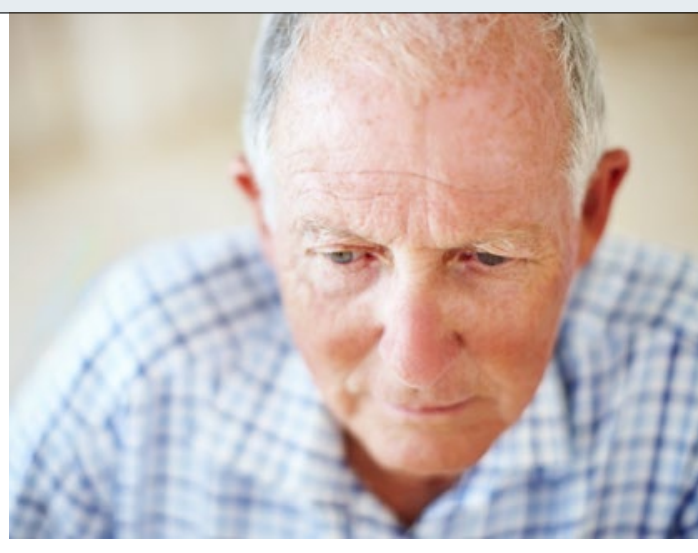

Depressivität beeinflusste die Mortalität.

mit Tumoren in späten Stadien (HR 1,32; $p=0,025)$ mit einer erhöhten Mortalität assoziiert.

Fazit: Bei Patienten mit Lungenkrebs haben longitudinale Veränderungen der Depressionssymptomatik Einfluss auf die Mortalität. Patienten mit Remission der Symptome haben eine vergleichbare Mortalität wie Patienten, die niemals depressiv waren. Diese Ergebnisse unterstreichen die Bedeutung einer antidepressiven Therapie bei Krebspatienten.

Judith Neumaier

Sullivan DR et al. Longitudinal Changes in Depression Symptoms and Survival Among Patients With Lung Cancer: A National Cohort Assessment. J Clin Oncol. 2016;34(33):3984-91.

\section{NSCLC: Intensitätsmodulierte Bestrahlung bewährt sich}

\begin{abstract}
Lokal fortgeschrittene nichtkleinzellige Lungenkarzinome (NSCLC) werden zunehmend häufiger intensitätsmoduliert bestrahlt. Welche Effekte erzielt diese im Vergleich zu einer dreidimensionalen konformalen Bestrahlung?
\end{abstract}

n einer sekundären Analyse der pros-
pektiven Studie RTOG 0617 wurden
intensitätsmodulierte Radiotherapie
(IMRT) und dreidimensionale konfor-
male Bestrahlung (3D-CRT) bei Patien-
ten mit lokal fortgeschrittenem NSCLC
verglichen. Diese hatten eine Chemothe-
rapie mit oder ohne Cetuximab sowie
gleichzeitig eine Bestrahlung mit 60
oder 74 Gy erhalten, $53 \%$ der 482 Pati-
enten mit 3D-CRT, $47 \%$ mit IMRT. Zwi-
schen den Gruppen bestanden deutliche
Unterschiede hinsichtlich der Dosime-
trie und Zielvolumina. Die IMRT-Grup-
pe hatte signifikant größere geplante Be-
strahlungsvolumina (median 486 vs. 427 $\mathrm{ml} ; \mathrm{p}=0,005)$, ein höheres Verhältnis von geplantem Bestrahlungsvolumen zu Lungenvolumen (median 0,15 vs. 0,13 ; $\mathrm{p}=0,013)$ und häufiger ein Stadium IIIB (38,6 vs. $30,3 \% ; \mathrm{p}=0,056)$.

Bei den 2-Jahres-Raten zu Gesamtüberleben und progressionsfreiem Überleben, lokalem Therapieversagen und Überleben ohne Fernmetastasen gab es keine signifikanten Unterschiede. Unter der IMRT kam es seltener zu Pneumonitis vom $\mathrm{Grad} \geq 3$ (3,5 vs. 7,9\%; $\mathrm{p}=0,039)$. Die Raten an Ösophagitis, Dysphagie, Gewichtsverlust und kardiovaskuläre Toxizitäten vom Grad $\geq 3$ waren in beiden Gruppen vergleichbar ( $p>0,05)$.
Die bestrahlten Herzvolumina waren bei der IMRT signifikant kleiner $(\mathrm{p}<0,05)$, obwohl sich die geplanten Bestrahlungsvolumina nicht unterschieden. Das mit 40 Gy bestrahlte Herzvolumen (V40) war signifikant mit dem Gesamtüberleben assoziiert $(\mathrm{p}<0,05)$, das mit 20 Gy bestrahlte Lungenvolumen (V20) signifikant mit einem erhöhten Risiko einer Pneumonitis vom Grad $\geq 3(p=0,026)$.

Fazit: Unter IMRT gab es weniger schwere Pneumonitiden und geringere kardiale Dosen. Die Forscher empfehlen daher den routinemäßigen Einsatz bei lokal fortgeschrittenem NSCLC. Judith Neumaier

Chun SG et al. Impact of Intensity-Modulated Radiation Therapy Technique for Locally Advanced Non-Small-Cell Lung Cancer: A Secondary Analysis of the NRG Oncology RTOG 0617 Randomized Clinical Trial. J Clin Oncol. 2017;35(1):56-62. 